\title{
GEOQUÍMICA DE FÓSFORO COMO INDICADORA DA QUALIDADE AMBIENTAL E DOS PROCESSOS ESTUARINOS DO RIO JAGUARIBE - COSTA NORDESTE ORIENTAL BRASILEIRA
}

\author{
Rozane Valente Marins*, Francisco José de Paula Filho e Carlos Artur Sobreira Rocha \\ Instituto de Ciências do Mar, Universidade Federal do Ceará, Av. da Abolição, 3207, 60125-120 Fortaleza - CE, Brasil
}

Recebido em 17/7/06; aceito em 18/12/06; publicado na web em 30/7/07

\begin{abstract}
PHOSPHORUS GEOCHEMISTRY AS A PROXY OF ENVIRONMENTAL ESTUARINE PROCESSES AT THE JAGUARIBE RIVER, NORTHEASTERN BRAZIL. Sedimentation of different phosphorus geochemical fractions can characterize the natural or anthropogenic processes dominant in the watershed. Selective chemical extraction of different phosphorus geochemical forms in estuarine sediments showed the predominance of inorganic over organic forms suggesting an increase in inorganic phosphorus input from anthropogenic sources. Local hydrochemistry favors the dominance of inorganic ferric and carbonatic phosphorus. Ongoing changes in the estuarine throphy, from mesothrophic to euthrophic, may decrease the immobilization of these forms, increasing dissolved phosphorus and favoring euthrophy. Detritic phosphorus suggests a fluvial origin of this fraction and acts as a tracer of river influence upon the estuary.
\end{abstract}

Keywords: environmental proxy; phosphorus; sediments

\section{INTRODUÇÃO}

Nos últimos anos, em virtude do acelerado crescimento urbano-industrial das cidades, tem aumentado substancialmente o aporte de cargas poluentes para os ecossistemas aquáticos costeiros, como lagoas, lagos e estuários ${ }^{1,2}$, que são ecossistemas de grande relevância para a manutenção de diversas espécies de animais e plantas. Os efeitos deletérios ocasionados pelo aporte de poluentes comprometem a qualidade ambiental dos ecossistemas ${ }^{3}$, tornando necessário o uso de indicadores e/ou índices para avaliação dessas alterações. Há pouco uso desses indicadores e índices para climas tropicais, dificultando a avaliação dos ecossistemas, por exemplo, da costa brasileira ${ }^{4}$.

Os estuários servem como filtros de materiais continentais em direção ao mar, atuando como zonas de deposição para alguns compostos químicos. No entanto, é importante salientar que estes podem não ser depósitos definitivos, em virtude de alterações geoquímicas do meio sedimentar ou aquático, ou como resposta a processos erosivos que resultam em remobilizações dos materiais depositados $^{5}$.

Dentre estes materiais, encontra-se o fósforo que é um nutriente essencial para a manutenção da vida, fazendo parte de diversas moléculas dos organismos vivos (e.g. ATP, ácidos nucléicos, fosfolipídios). Este elemento é, também, considerado um nutriente limitante para a produção primária das células fitoplanctônicas dos sistemas aquáticos $\operatorname{costeiros}^{6,7} \mathrm{e}$ tem sido considerado como principal responsável pela eutrofização artificial em águas continentais ${ }^{8}$.

O fósforo presente ou emitido para os estuários por fontes naturais ou antrópicas pode ser de origem orgânica ou inorgânica.

São fontes naturais significativas de fósforo o intemperismo das rochas fosfáticas (apatita detrítica), as chuvas e a lixiviação dos solos pelas águas das chuvas, sendo que a fluorapatita carbonática autigênica também representa um importante depósito em sedimentos marinhos para o fósforo reativo presente em águas oceânicas. Por outro lado, a fração orgânica de fósforo encontrada em sedimentos estuarinos é resultado da contribuição de diferentes

*e-mail: rmarins@labomar.ufc.br fontes; sua variabilidade de composição dificulta a identificação e quantificação de seus constituintes. Além disto, o fósforo orgânico é constantemente modificado em sua essência pelos processos de degradação em trânsito na interface água/sedimento? .

O fósforo de origem antrópica encontra-se principalmente nas formas inorgânicas, devido ao uso indiscriminado de fertilizantes químicos nos solos, como o diidrogenofosfato de cálcio $\mathrm{Ca}\left(\mathrm{H}_{2} \mathrm{PO}_{4}\right)_{2}$, altamente solúvel em água, ou pelas emissões de efluentes urbanos, que contêm polifosfatos dos produtos de limpeza e ortofosfato e fosfatos inorgânicos condensados dos esgotamentos sanitários não $\operatorname{tratados}^{10}$.

Ao serem carreados pelos rios, os materiais fosfatados podem permanecer em meio aquoso ou serem depositados nos sedimentos, sob diferentes formas, que irão descrever de forma integrada os processos antrópicos que influenciam as bacias hidrográficas e, possivelmente, processos naturais que caracterizam, por ex., a hidrogeoquímica local, visto que interfere nos processos biogeoquímicos predominantes e na sedimentação de materiais ${ }^{11-13}$.

As águas estuarinas da costa nordeste oriental brasileira possuem elevados níveis de oxigênio, $\mathrm{pH}$ alcalino, bem como concentrações significativas de carbonatos e oxi-hidróxidos de ferro ${ }^{14}$ que favorecem a manutenção do fósforo na fase sólida ${ }^{15-19}$. Entretanto, estes fatores podem ser alterados localmente, por exemplo, pela presença de barragens nos rios da região. Como consequiência, nos estuários desses rios fortemente açudados ocorre a forte penetração de águas marinhas, como no caso do Rio Jaguaribe, Ceará, onde a cunha salina durante períodos de seca somente é barrada pela presença de um dique a $34 \mathrm{~km}$ da foz do rio ${ }^{20,21}$. Além disso, no caso do rio Jaguaribe, o crescimento urbano recente tem contribuído para o aporte diferenciado dos materiais fosfatados ${ }^{22}$.

Estimativas das cargas de fósforo para o estuário do rio Jaguaribe indicam que os esgotos urbanos representam a maior fonte deste elemento para a região, cerca de $39 \%$ das emissões totais do elemento, enquanto que a pecuária emite cerca de $34 \%$ e a carcinicultura e a agricultura contribuem, respectivamente, com 11 e $4 \%$ do total das cargas emitidas de fósforo. $\mathrm{O}$ intemperismo dos solos locais representa a principal fonte natural do elemento para este ecossistema, contribuindo com cerca de $13 \%$ das emissões totais ${ }^{23}$. 
Assim, neste estudo, a distribuição e partição geoquímica de fósforo em sedimentos superficiais foram avaliadas como indicadoras da qualidade ambiental e dos processos estuarinos predominantes ao longo do estuário do rio Jaguaribe, em duas ocasiões, para elucidar qual a predominância atual das fontes naturais e antrópicas de fósforo. Este objetivo foi alcançado através de medidas dos teores totais de fósforo e dos teores das diferentes frações geoquímicas às quais o elemento está associado no sedimento, através do uso de extração química seletiva estabelecida para as diferentes formas inorgânica e orgânica de fósforo.

\section{PARTE EXPERIMENTAL}

\section{Área de estudo e amostragem}

A bacia do rio Jaguaribe está inserida na zona de domínio do clima semi-árido. Este clima apresenta dois períodos significativamente distintos, sendo um longo e seco, com duração média de 8 meses por ano, e um curto conhecido como quadra invernosa, chuvoso e irregular. A prolongada ausência das chuvas, mais de 8 meses sem chuva, caracteriza a "seca" na região ${ }^{24}$. As temperaturas são elevadas com reduzidas amplitudes, sendo a média anual na faixa de $26,9^{\circ} \mathrm{C}$, o que pode favorecer o metabolismo do ecossistema costeiro local.

Os grandes açudes da bacia do rio Jaguaribe, como o Castanhão e Orós, são responsáveis por $70 \%$ do volume total represado de suas águas. A açudagem tem sido caracterizada como o fator de maior impacto para a zona costeira devido à intensificação dos baixos deflúvios do rio e do aporte de sedimentos para a zona costeira adjacente ${ }^{25}$. Por ex., em épocas de estiagem, a região estuarina adentra o continente até $34 \mathrm{~km}$ da linha de costa e, como consequiência desta dinâmica, o estuário do rio é do tipo bem misturado, entretanto nos canais secundários tem sido constatada estratificação química com valores de oxigênio decaindo a $<4,00 \mathrm{mg} / \mathrm{L}$, a profundidade de $6 \mathrm{~m}$, o que favorece os processos anaeróbicos ${ }^{14}$. Estas características da hidroquímica local ${ }^{20,21}$, muito provavelmente, também intensificam os processos sazonais que dominam a dinâmica do fósforo na região costeira.

Dessa forma, para avaliar as diferenciações na distribuição e partição do fósforo, ao longo do estuário do rio Jaguaribe, foram realizadas duas campanhas de amostragem, para coleta de sedimentos superficiais (em novembro de 2001 e outubro de 2003).

As estações de coleta ao longo da região de estudo são apresentadas na Figura 1 e descritos na Tabela 1.

As amostras de sedimentos superficiais foram coletadas na margem do canal principal, permanentemente submersa, utilizando-se pá de plástico e acondicionadas em sacos plásticos. Em cada ponto foram coletadas três amostras e compostas por ponto, utilizando-se protocolos limpos ${ }^{26}$. As amostras foram acondicionadas sob refrigeração até o laboratório, onde foram secas em estufa à $60{ }^{\circ} \mathrm{C}$ e, em seguida, peneiradas mecanicamente para obtenção da fração granulométrica $<63 \mu \mathrm{m}$, para minimizar o efeito da granulometria dos sedimentos sobre a distribuição de fósforo ${ }^{7,9}$.

\section{Determinação da partição geoquímica de fósforo nos sedimentos estuarinos}

Os métodos de extração seqüencial são operacionalmente definidos com base na reatividade da fração que se deseja extrair, espécie alvo, em relação a uma solução extratora conhecida9. Neste trabalho foi utilizado o esquema de extração estabelecido por Berner e $\mathrm{Rao}^{7}$ que permite a separação de seis fases distintas de fósforo (Figura 2), de forma a determinar a origem do fósforo contido nos sedi-

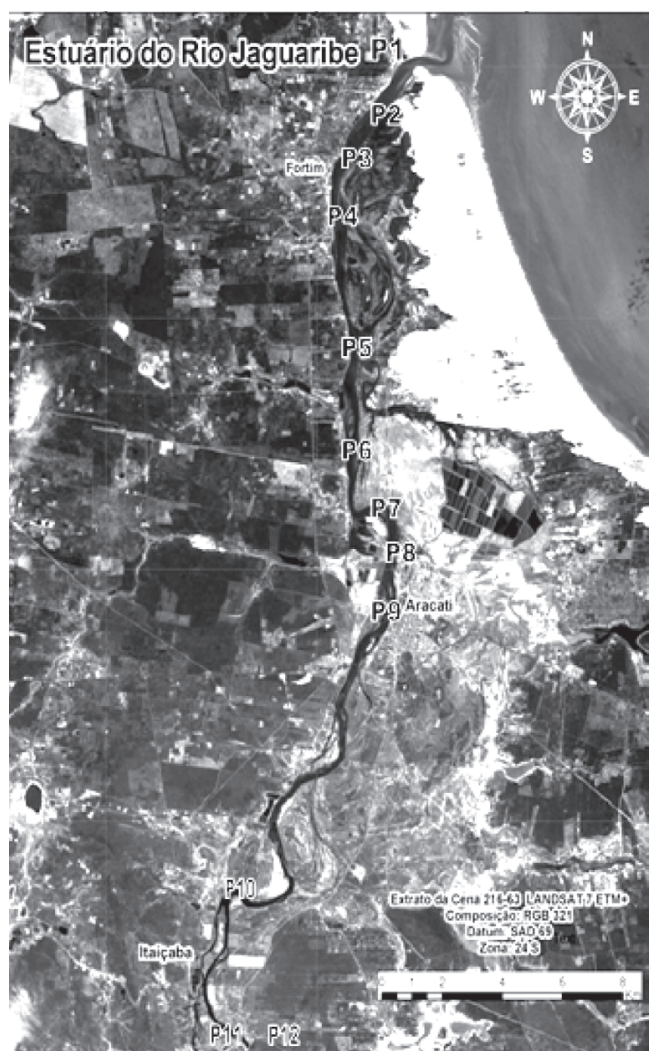

Figura 1. Imagem de satélite Landsat da área de estudo compreendendo a porção estuarina do Rio Jaguaribe/CE

Tabela 1. Descrição dos estações de amostragem relacionadas à Figura 1

\begin{tabular}{lc}
\hline Estação de coleta & Localidade \\
\hline P1 & Foz (margem esquerda) \\
P2 & Foz (margem direita) \\
P3 & Canal do Amor \\
P4 & Ilha do Caldeleiro \\
P5 & Jardim Alto \\
P6 & Ilha dos Veados \\
P7 & Aracati jusante \\
P8 & Aracati \\
P9 & Ponte da Br - 304 \\
P10 & Cabreiro \\
P11 & Itaiçaba jusante da passagem \\
P12 & Itaiçaba montante da passagem \\
\hline
\end{tabular}

\begin{tabular}{|c|c|}
\hline $\begin{array}{c}\text { FÓSFORO TOTAL } \\
\text { total das frações inorgânicas e orgânicas }\end{array}$ \\
\hline $\begin{array}{c}\text { FÓSFORO TOTAL INORGÂNICO } \\
\text { total das frações de P } \\
\text { ligadas ao Fe, CaCO3, trocável } \\
\text { e detrítico. }\end{array}$ & $\begin{array}{c}\text { FÓSFORO TOTAL ORGÂNICO } \\
\text { frações de fósforo adsorvidas } \\
\text { por subst. húmicas, resíduos orgânicos, } \\
\text { plâncton e etc. }\end{array}$ \\
\hline $\begin{array}{c}\text { FÓSFORO LIGADO A Fe } \\
\text { fração do P fortemente ligada } \\
\text { a oxi-hidróxidos de Fe }\end{array}$ & $\begin{array}{c}\text { FÓSFORO ACÉTICO } \\
\text { fração do P ligada aos } \\
\text { carbonatos }\end{array}$ \\
\hline
\end{tabular}

Figura 2. Resumo das frações de fósforo determinadas neste estudo de acordo com a metodologia de Berner e Rao ${ }^{6}$

mentos bem como a qualidade desses sedimentos. Neste procedimento, três subamostras de sedimentos superficiais foram analisa- 


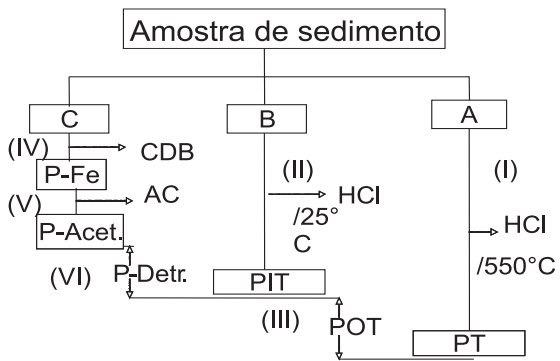

Figura 3. Esquema de extração seqüencial seletiva de fósforo em sedimentos, adaptado de Berner e Rao ${ }^{6}$. (I) - Alíquota A - queima à $550{ }^{\circ} \mathrm{C}$, por $12 \mathrm{~h}$, seguida de extração com $\mathrm{HCl}$ 1,0 M, por 16 h. (II) Alíquota B - lixiviação nas mesmas condições de extração (I), sem combustão prévia. (III) Determinação indireta de POT por diferença (a matéria orgânica não é atacada pelo $\mathrm{HCl}$ ). (IV) - Extração seletiva de P-Fe com mistura citratoditionito-bicarbonato $(C D B), p H \approx 7,6$, por $8 h .(V)$ - Extração subseqüente do resíduo de (IV) em solução tampão pH 4,0 (NaAc/HAc), durante 6 h. VIDeterminação indireta de P-Detr. $=$ PIT- $(P-F e+P$-Acet . $)$. Todas as extrações foram efetuadas sob agitação à temperatura ambiente

das em separado para cada uma das etapas de extração (Figura 3).

A primeira subamostra foi utilizada na determinação de fósforo total (PT) através da queima do sedimento, à $550{ }^{\circ} \mathrm{C}$, por $12 \mathrm{~h}$. Após a queima o resíduo foi levado à digestão em solução de $\mathrm{HCl}$ 1,0 M, sob agitação.

Para determinação do fósforo inorgânico total (PIT) a segunda subamostra não levada à combustão foi lixiviada diretamente por solução de $\mathrm{HCl} 1,0 \mathrm{M}$, à temperatura ambiente e sob constante agitação. A concentração de fósforo orgânico total (POT) foi obtida por diferença entre as concentrações de PT e PIT. A técnica de Berner e $\mathrm{Rao}^{7}$ reduz a perda de material que ocorre em outras metodologias onde as extrações são seqüenciais ${ }^{2,9,19,27-31}$.

A terceira subamostra foi complementarmente usada para a partição do fósforo inorgânico total (PIT).

A partição do PIT (Figura 3) foi medida para as frações fósforo ligado fortemente aos oxi-hidróxidos de ferro (P-Fe), à fluorapatita carbonática de origem autigênica, mais apatita biogênica e à $\mathrm{CaCO}_{3}$ (P-Acet). Uma terceira fração foi obtida de forma indireta, por diferença entre o PIT e a soma do P-Fe com o P-Acet, que determinou a apatita detrítica de origem ígnea ou metamórfica (P-Detr).

A extração da fração P-Fe foi realizada utilizando-se como solução extratora a mistura citrato-ditionito-bicarbonato (CDB), a temperatura ambiente e sob agitação constante, que é considerada seletiva à fase férrica do fósforo e a alguma fração de fósforo reativo, mantido o $\mathrm{pH}$ próximo à neutralidade, $\mathrm{pH} 7,6$, que é fundamental para a efetiva seletividade desta extração?.

A fração P-Acet. foi obtida a partir da extração do resíduo da fração P-Fe utilizando-se solução tampão de acetato de sódio/ácido acético a pH 4,0, sob agitação, a temperatura ambiente a $^{7,9,16}$.

Resumindo, para as amostras de sedimento de cada estação de coleta foram realizadas análises para obtenção do fósforo total (PT) e das frações de fósforo inorgânico (PIT), fósforo ligado a oxihidróxidos de ferro (P-Fe) e fósforo carbonático (P-Acet). De forma indireta, foram obtidas a fração detrítica (P-Detr.) e o fósforo orgânico total (POT).

Ao final das diferentes extrações a espécie resultante foi totalizada como ortofosfato através da espectrofotometria na região do visível, utilizando-se a metodologia do azul de molibdato e sucessivas diluições da solução padrão de diidrogeno fosfato de potássio, contendo $10 \mathrm{mmol} / \mathrm{L}$ de fosfato para a preparação de padrões para a calibração do equipamento ${ }^{26}$.

Excepcionalmente, para a determinação da fração P-Fe foi empregada metodologia de detecção final alternativa, em virtude da solução extratora de CDB interferir fortemente com o molibdato de amônio da metodologia tradicional ${ }^{26}$. Neste procedimento alternativo o extrato é pré-tratado com uma solução de cloreto férrico $1 \%$, a fim de eliminar o efeito do citrato sobre a detecção do complexo azul de fosforomolibdato ${ }^{32}$.

Adicionalmente às medidas de fósforo foram determinados os teores dos carreadores geoquímicos que controlam a retenção e a mobilidade de contaminantes em solos e sedimentos: matéria orgânica total e facilmente oxidável, ferro total e carbonato ${ }^{33,34}$.

\section{RESULTADOS E DISCUSSÃO}

\section{Distribuição e partição geoquímica de fósforo como indicadoras ambientais no estuário do Rio Jaguaribe}

O limite de detecção da técnica de medição de fósforo, na forma de ortofosfato, foi considerado igual a três vezes o desvio estimado por Sy/x dividido pela sensibilidade da reta, a partir da reta de regressão obtida da curva de calibração, assumindo-se que no método dos mínimos quadrados não ponderados, cada ponto do gráfico (incluído o ponto representativo do branco) tem uma variação normalmente distribuída (somente na direção de y) ${ }^{35}$. A média dos limites determinados ao longo do estudo, para as curvas elaboradas em diferentes dias para a faixa de 1 a $7 \mu \mathrm{mol} \mathrm{L} \mathrm{L}^{-1}$, foi de $0,2 \mu \mathrm{mol} \mathrm{L}{ }^{-1}$, similar aos limites relatados na literatura ${ }^{26}$.

A validação dos resultados obtidos para a determinação do fósforo total contido em sedimentos, na forma de ortofosfato, foi feita a partir da determinação em paralelo de material certificado NIST referência 1646a ("National Institute of Standards \& Technology"). A recuperação média do teor de fósforo total $270 \pm 0,001 \mu \mathrm{g} \mathrm{g}^{-1}$, única fração referenciada em padrão de sedimento estuarino, foi de $91,9 \pm 8,3 \%$ do valor certificado.

A distribuição dos teores médios do fósforo total (PT) ao longo do estuário do rio Jaguaribe, e respectivos desvios padrões, são apresentados na Tabela 2.

Tabela 2. Resultados de fósforo total (PT), em $\mu \mathrm{g} \mathrm{g}^{-1}$, ao longo do estuário do rio Jaguaribe (CE), para as n determinações realizadas para as diferentes amostras compostas a partir das triplicatas coletadas por ponto

\begin{tabular}{lcc}
\hline Estações de coleta & $1^{\mathrm{a}}$ campanha $(\mathrm{n})$ & $2^{\mathrm{a}}$ campanha $(\mathrm{n})$ \\
\hline P1 & $101,9 \pm 22,0(8)$ & $(0)$ \\
P2 & $95,1 \pm 19,3(8)$ & $107,3 \pm 0,5(4)$ \\
P3 & $(0)$ & $125,8 \pm 2,4(4)$ \\
P4 & $112,2 \pm 26,7(8)$ & $96,0 \pm 0,9(4)$ \\
P5 & $110,5 \pm 12,4(8)$ & $106,1 \pm 2,8(4)$ \\
P6 & $85,6 \pm 29,0(8)$ & $122,5 \pm 4,6(4)$ \\
P7 & $100,5 \pm 5,7(8)$ & $(0)$ \\
P8 & $(0)$ & $157,1 \pm 3,2(4)$ \\
P9 & $(0)$ & $87,1 \pm 12,7(4)$ \\
P10 & $92,7 \pm 9,6(8)$ & $83,9 \pm 3,6(4)$ \\
P11 & $82,7 \pm 4,5(2)$ & $77,6 \pm 6,9(4)$ \\
P12 & $88,7 \pm 2,1(2)$ & $77,5 \pm 11,4(4)$ \\
\hline
\end{tabular}

As concentrações de fósforo total ao longo do estuário do rio Jaguaribe permaneceram similares entre a primeira e a segunda campanha, variando na faixa de 77,5 $\pm 11,4 \mu \mathrm{g} \mathrm{g}^{-1}(\mathrm{P} 12)$ a $157,1 \pm$ $3,2 \mu \mathrm{g} \mathrm{g}^{-1}$ (P8) (sendo $\mathrm{n}=8$, exceto para P11 e P12 onde $\mathrm{n}$ foi igual a 2, na primeira campanha, e $\mathrm{n}$ igual a 4 para todas as estações da segunda campanha), mostrando uma tendência de acúmulo de fósforo nos sedimentos a partir de P8 em direção a foz. 
Para verificar a homogeneidade da distribuição espacial do PT ao longo de todo o estuário foram agrupados e analisados através da ANOVA de fator único para $\alpha=0,05$, os dois conjuntos de médias obtidos para o fósforo total nas duas campanhas realizadas.

Com o resultado do teste foi identificada a existência de diferenciação estatística entre as médias, e a partir desta constatação foi aplicado o teste de Tukey, $\alpha=0,05$, para especificar quais médias são estatisticamente diferenciadas das demais espacialmente ao longo do estuário. A análise dos dados revelou que o ponto P8 é o mais distinto e está localizado em Aracati (Figura 1 e Tabela 1). Esta diferenciação provavelmente se deve a maior retenção de fósforo total nesta região do estuário, devido à retenção hídrica que ocorre em maior extensão durante o período de seca regional. Durante este período, o tempo de residência das águas estuarinas pode atingir $13 \operatorname{dias}^{20,21}$. Ou, esta diferenciação do ponto P8 em relação aos demais, pode também estar refletindo maior aporte de fósforo total em Aracati (maior município da região com áreas significativas de uso para carcinicultura).

Para interpretação da partição geoquímica de fósforo foram determinados os teores dos carreadores geoquímicos. Os resultados dos teores nos sedimentos amostrados de matéria orgânica, matéria orgânica facilmente oxidável, ferro total e carbonatos estão apresentados na Tabela 3 e serão discutidos junto aos resultados de fósforo para verificação dos processos geoquímicos relevantes.

A partição geoquímica do fósforo total (PT) nos sedimentos nas frações fósforo inorgânico total (PIT) e orgânico total (POT) mostrou que, embora não tenha havido aumento significativo dos teores de PT, durante o período das campanhas realizadas, houve um aumento dos teores de PIT nos sedimentos na segunda campanha. As concentrações de fósforo inorgânico total variaram na primeira campanha entre 43,0 \pm 7,0 $\mu \mathrm{g} \mathrm{g}^{-1}(\mathrm{P} 6)$ a $68,1 \pm 5,9 \mu \mathrm{g} \mathrm{g}^{-1}(\mathrm{P} 4)$ $(\mathrm{n}=6$, exceto para $\mathrm{P} 11$ e P12 onde $\mathrm{n}=2$ ) enquanto que os valores de fósforo orgânico total variaram de 25,5 (P11) a 58,1 (P1) $\mu \mathrm{g} \mathrm{g} \mathrm{g}^{-1}$. $\mathrm{Na}$ segunda campanha as concentrações de fósforo inorgânico total (PIT) variaram de 57,6 $\pm 0,7 \mu \mathrm{g} \mathrm{g}^{-1}$ (P12) a $124,8 \pm 1,2 \mu \mathrm{g} \mathrm{g}^{-1}$ (P8), sendo $\mathrm{n}=4$ para todas as estações, e os resultados de POT variaram de 10,4 (P11) a $32,3 \mu \mathrm{g} \mathrm{g}^{-1}(\mathrm{P} 8)$.

Os percentuais das frações PIT e POT apresentados na Figura
4 mostram que a fração orgânica sempre apresentou resultados inferiores às de fósforo inorgânico, exceto na região da foz (P1), onde a área de mangues é a mais representativa de todo o estuário. Durante a segunda campanha, para as mesmas estações de coleta da primeira campanha, houve um aumento do predomínio das frações inorgânicas de fósforo que atingiu valores superiores a $86 \%$ do fósforo total, caracterizando um aumento do aporte dessa fração geoquímica para os sedimentos estuarinos.

Embora a costa nordeste oriental seja conhecida como oligotrófica, estes resultados mostram que a retenção hídrica local, provavelmente potencializada pelo aumento do aporte antrópico de fósforo inorgânico, pode levar particularmente esta região estuarina a um acúmulo de fósforo inorgânico em sedimentos.

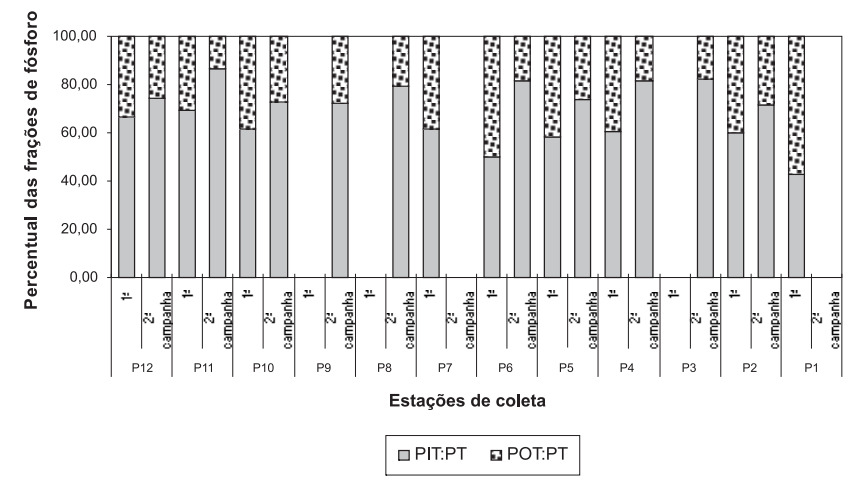

Figura 4. Partição geoquímica do fósforo em sedimentos superficiais do estuário do Rio Jaguaribe (CE) ( $1^{a}$ campanha = coleta de novembro de 2001 e $2^{a}$ campanha $=$ coleta de outubro de 2003)

Corroborando as conclusões de que o aporte de fósforo foi modificado entre os períodos da amostragem observou-se que durante a primeira campanha os carreadores geoquímicos (matéria orgânica total e facilmente oxidável) apresentaram significativa correlação estatística com as frações geoquímicas de fósforo. Considerando-se $n=9$ e $\alpha=0,05$, foram significativas as correlações obtidas entre as frações PT e a matéria orgânica total $(r=0,601)$ e

Tabela 3. Teores dos carreadores geoquímicos principais determinados nos sedimentos amostrados para avaliação dos processos retentores de fósforo em sedimentos

\begin{tabular}{|c|c|c|c|c|c|c|}
\hline Estações de coleta & M.O. Total (\%) & M.O. oxidável (\%) & Ferro Total (\%) & Carbonatos $(\%)$ & Salinidade & $\mathrm{O}_{2}$ dissolvido $\mathrm{mg} \mathrm{L}^{-1}$ \\
\hline $\mathrm{P} 1$ & $9,5 \pm 0,4$ & $2,5 \pm 0,1$ & $4,0 \pm 0,1$ & & 38,10 & 5,95 \\
\hline $\mathrm{P} 2^{\mathrm{a}}$ & $1,0 \pm 0,1$ & $0,7 \pm 0,1$ & $1,8 \pm 0,1$ & & 38,30 & 53,68 \\
\hline $\mathrm{P} 2^{\mathrm{b}}$ & $2,5 \pm 0,1$ & $0,6 \pm 0,1$ & $2,0 \pm 0,1$ & $23,7 \pm 2,1$ & 37,70 & 7,50 \\
\hline P3 & $3,3 \pm 0,3$ & $0,8 \pm 0,1$ & $3,8 \pm 0,3$ & $72,5 \pm 8,4$ & 38,00 & 7,12 \\
\hline $\mathrm{P} 4^{\mathrm{a}}$ & $3,2 \pm 0,1$ & $0,8 \pm 0,1$ & $2,6 \pm 0,1$ & & 39,00 & 5,64 \\
\hline $\mathrm{P} 4^{\mathrm{b}}$ & $5,4 \pm 0,2$ & $1,2 \pm 0,1$ & $7,0 \pm 0,4$ & $82,4 \pm 3,4$ & 38,30 & 7,26 \\
\hline $\mathrm{P} 5^{\mathrm{a}}$ & $6,4 \pm 0,2$ & $1,1 \pm 0,1$ & $2,4 \pm 0,1$ & & 39,10 & 5,19 \\
\hline $\mathrm{P} 5^{\mathrm{b}}$ & $6,8 \pm 0,2$ & $2,7 \pm 0,2$ & $8,6 \pm 0,2$ & $59,1 \pm 4,4$ & 38,10 & 7,17 \\
\hline$P 6^{\mathrm{a}}$ & $2,6 \pm 0,1$ & $0,7 \pm 0,1$ & $2,7 \pm 0,1$ & & 39,10 & 5,75 \\
\hline $\mathrm{P} 6^{\mathrm{b}}$ & $8,2 \pm 0,1$ & $1,4 \pm 0,1$ & $14,2 \pm 0,5$ & $72,4 \pm 3,4$ & 38,00 & 9,23 \\
\hline P7 & $3,7 \pm 0,1$ & $0,7 \pm 0,1$ & $3,1 \pm 0,1$ & & 39,10 & 5,26 \\
\hline P8 & $3,7 \pm 0,1$ & $1,9 \pm 0,1$ & $15,0 \pm 0,6$ & $43,6 \pm 2,3$ & 35,40 & 8,88 \\
\hline P9 & $3,7 \pm 0,4$ & $1,0 \pm 0,1$ & $6,1 \pm 0,3$ & $42,4 \pm 3,5$ & 33,10 & 8,22 \\
\hline $\mathrm{P} 10^{\mathrm{a}}$ & $2,6 \pm 0,1$ & $0,9 \pm 0,1$ & $3,3 \pm 0,1$ & & 36,50 & 8,40 \\
\hline $\mathrm{P} 10^{\mathrm{b}}$ & $6,3 \pm 0,1$ & $3,2 \pm 0,1$ & $8,5 \pm 0,7$ & $20,9 \pm 1,8$ & 27,40 & 5,98 \\
\hline $\mathrm{P} 11^{\mathrm{a}}$ & $0,6 \pm 0,1$ & $0,1 \pm 0,1$ & $2,0 \pm 0,1$ & & 29,40 & 5,61 \\
\hline $\mathrm{P} 11^{\mathrm{b}}$ & $1,8 \pm 0,1$ & $0,6 \pm 0,1$ & $5,5 \pm 0,1$ & $12,8 \pm 2,1$ & 21,90 & 5,83 \\
\hline $\mathrm{P} 12^{\mathrm{a}}$ & $1,3 \pm 0,4$ & $0,3 \pm 0,1$ & $2,0 \pm 0,1$ & & 0,40 & 8,40 \\
\hline $\mathrm{P} 12^{\mathrm{b}}$ & $2,3 \pm 0,1$ & $0,8 \pm 0,1$ & $6,4 \pm 0,1$ & $15,6 \pm 2,0$ & 0,40 & 7,79 \\
\hline
\end{tabular}

(a) Campanha de 2001 (b) Campanha de 2003 
POT e a matéria orgânica total e a facilmente oxidável $(r=0,893$ e $r=0,918$, respectivamente). Entretanto, durante a segunda campanha somente foram encontradas correlações significativas das frações geoquímicas de PT e PIT com o ferro $(r=0,543$ e $r=0,557$, respectivamente, para $\mathrm{n}=10$ e $\alpha=0,05$ ).

Por outro lado, as correlações de fósforo total e inorgânico total com parâmetros hidroquímicos tais como a salinidade e o oxigênio dissolvido foram significativas somente na segunda campanha (para PT, $r=0,575$ e $r=0,912$ e para PIT, $r=0,547$ e 0,740, sendo $n=10$ e $\alpha=0,05)$, caracterizando a dependência das precipitações sedimentares e, conseqüentemente, a retirada de fósforo total e inorgânico da coluna d'água para os sedimentos, das características intrínsecas da dinâmica da própria coluna d'água.

\section{Distribuição e partição do fósforo inorgânico}

Complementando a avaliação da origem do aumento da fração PIT em sedimentos foram determinadas as concentrações das três frações inorgânicas (P-Fe; P-Acet. e P-Detr.) em quadruplicatas das amostras compostas por ponto de coleta da campanha de novembro de 2001 e, em sextuplicata da campanha de outubro de 2003. Os teores das diferentes frações de fósforo inorgânico são apresentados nas Figuras 5 e 6.

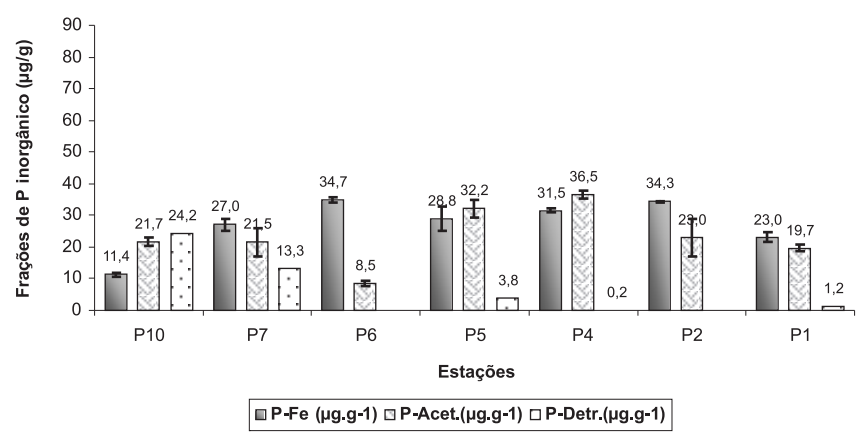

Figura 5. Distribuição e teores médios (para $n=4$ ) das diferentes frações inorgânicas do fósforo em sedimentos superficiais do estuário do Rio Jaguaribe/CE (novembro de 2001)

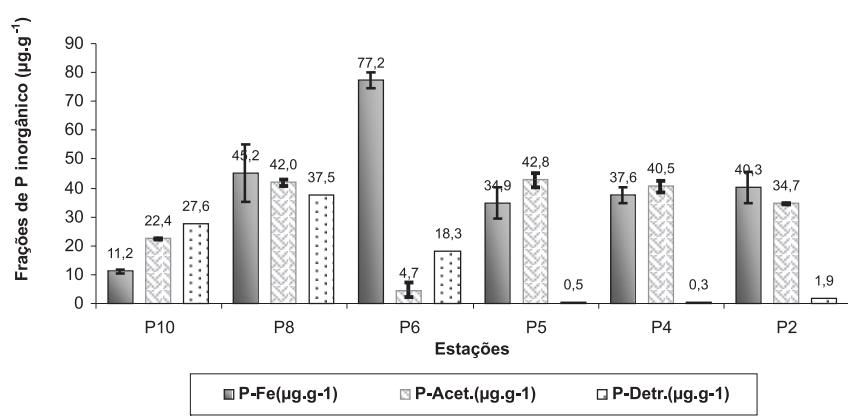

Figura 6. Distribuição e teores médios (para $n=6$ ) das frações inorgânicas do fósforo em sedimentos superficiais do estuário do Rio Jaguaribe/CE (outubro de 2003)

Na primeira campanha as concentrações da fração fósforo ligado a oxi-hidróxidos de ferro (P-Fe) variaram de 11,4 $\pm 5,4 \mu \mathrm{g} \mathrm{g}^{-1}$ (P10) a $34,7 \pm 2,1 \mu \mathrm{g} \mathrm{g}^{-1}(\mathrm{P} 6)$, representando em média cerca de $30 \%$ do fósforo total e $50 \%$ do fósforo inorgânico total, correspondendo à fração predominante no sistema, embora tenha apresentado uma distribuição geográfica pouco definida ao longo do estuário.

A fração de fósforo associado a carbonatos (P-Acet.) variou de $8,5 \pm 0,7 \mu \mathrm{g} \mathrm{g}^{-1}$ (P6) a 36,5 $\pm 1,2 \mu \mathrm{g} \mathrm{g}^{-1}$ (P4). Esta fração, P-Acet. (apatita carbonática autigênica), representou a segunda maior fração do fósforo presente nos sedimentos superficiais, perfazendo em média $24 \%$ do conteúdo total de fósforo e $40 \%$ da fração inorgânica. Assim como a fração P-Fe, a fração P-Acet. apresentou uma distribuição geográfica pouco definida ao longo do estuário, entretanto sua presença nos sedimentos estuarinos do Rio Jaguaribe, em percentuais elevados, confirma que este sistema é atualmente predominantemente dominado por águas marinhas que enriquecidas em fósforo reativo contribuem para estas deposições sedimentares.

A fração apatita detrítica de origem ígnea ou metamórfica ( $\mathrm{P}$ Detr.) variou de não detectável (P2 e P6) a 24,2 $\mu \mathrm{g} \mathrm{g}^{-1}$ (P10) apresentando um forte decréscimo a partir de P7 (a jusante de Aracati). Os menores teores estão associados a esta fração que representou apenas $6 \%$ do fósforo total e cerca de $10 \%$ do fósforo inorgânico.

Os resultados de P-Detr. mostram que as deposições de apatita detrítica delimitam a zona de influência fluvial para o estuário, corroborando os estudos hidroquímicos realizados na região ${ }^{19,20} \mathrm{e}$ denotam esta fração geoquímica como um bom traçador da influência fluvial em estuários da costa nordeste oriental.

Os resultados obtidos para as três frações não apresentaram correlações significativas com os carreadores geoquímicos analisados, enquanto que em relação aos parâmetros hidroquímicos foram obtidas correlações significativas somente entre o P-Fe e a salinidade $(r=0,795)$ e P-Detr. e o oxigênio dissolvido $(r=0,663)$, em ambos os casos $n=7$ e $\alpha=0,05$.

Os teores obtidos para as diferentes frações inorgânicas do fósforo referentes à segunda campanha foram semelhantes aos da primeira (Figura 6). As concentrações das frações inorgânicas variaram de $11,2 \pm 0,6 \mu \mathrm{g} \mathrm{g}^{-1}(\mathrm{P} 10)$ a $77,2 \pm 2,7 \mu \mathrm{g} \mathrm{g}^{-1}$ (P6) para o fósforo ligado a oxi-hidróxidos de ferro (P-Fe). $\mathrm{O}$ fósforo associado a carbonatos (P-Acet.) variou de $22,4 \pm 0,1 \mu \mathrm{g} \mathrm{g}^{-1}$ (P6) a 42,8 \pm $2,5 \mu \mathrm{g} \mathrm{g}^{-1}$ (P5), e seguindo o comportamento da primeira campanha o P-Detr. apresentou valores insignificantes (de 0,3 a $37,5 \mu \mathrm{g}$ $\mathrm{g}^{-1}$ ) nas porções do médio e baixo estuário (P2, P4 e P5), sendo os valores mais expressivos na região superior do estuário (P6, P8 e P10) onde variaram de 18,3 a $38,5 \mu \mathrm{g} \mathrm{g}^{-1}$, novamente delimitando a zona de influência fluvial do estuário, corroborando os estudos hidroquímicos realizados na região ${ }^{19-20}$ e confirmando que esta fração geoquímica é útil como traçadora da influência fluvial em estuários da costa nordeste oriental.

Os teores de $\mathrm{P}-\mathrm{Fe}$ encontrados na segunda campanha representaram até $36 \%$ do fósforo total e $46 \%$ do fósforo inorgânico total, confirmando os resultados da primeira campanha, demonstrando que comparativamente às demais frações inorgânicas esta fração constituiu a maior fração inorgânica de fósforo presente nos sedimentos estuarinos do Rio Jaguaribe.

Correlações estatisticamente significativas foram encontradas entre o P-Fe e ferro $(\mathrm{r}=0,557)$ e o oxigênio $(\mathrm{r}=0,911)$ sendo $\mathrm{n}=$ $6, \alpha=0,05$, mostrando que a maior parte do fósforo inorgânico é retida no estuário associada a ferro e depende das condições óxicas dos sedimentos, confirmando os fluxos previstos de deposição sedimentar de fósforo em ambiente tropical ${ }^{8}$.

Os controles biogeoquímicos da dinâmica do fósforo em sistemas estuarinos têm sido extensivamente estudados ${ }^{17,30}$. Seu comportamento nos sedimentos muitas vezes é estreitamente associado com o ciclo redox do ferro. Em sedimentos estuarinos tropicais o ferro é um carreador geoquímico de grande relevância, principalmente em ambientes empobrecidos de matéria orgânica. $\mathrm{O}$ metal assume um papel chave no ciclo biogeoquímico do fósforo, visto que o fósforo ligado a ferro é um depósito dinâmico e potencialmente extenso para este nutriente. Desta maneira a disponibilidade de oxigênio na coluna d'água e nos sedimentos, e em especial na interface sedimento/água, repercute diretamente na formação diagenética de oxi-hidróxidos de ferro nos sedimentos ${ }^{18}$. 
A fração fósforo associado a carbonatos (P-Acet.) representou $29 \%$ do conteúdo total de fósforo e $38 \%$ da fração inorgânica. Foi encontrada correlação estatisticamente significativa entre as concentrações de P-Acet. e os carbonatos $(\mathrm{r}=0,700)$ bem como com a salinidade e o oxigênio dissolvidos $(r=0,877$ e $r=0,740$, respectivamente), em todos os casos para $n=6$ e $\alpha=0,05$. Os demais carreadores geoquímicos e parâmetros hidroquímicos analisados não apresentaram correlação significativa com o P-Acet., mostrando que a retenção hídrica do estuário que favorece a salinização de suas águas, também favoreceu a retenção de fósforo em sedimentos na fração inorgânica P-Acet nesta ocasião.

A fração carbonática ${ }^{11}$ representou até $56 \%$ de fósforo associado a cálcio no setor franja de lama e $58 \%$ no setor ecossistema manguezal, em Itacuruçá, RJ. No nordeste da Baía da Flórida, EUA, os valores são extremos para esta fração, chegando a compor até $96 \%$ da fração inorgânica e $56 \%$ da fração total de fósforo sedimentar ${ }^{18}$. Por outro lado, percentuais menores, variando entre 22 a 58,4\%, foram encontrados no estuário de Loire, na França ${ }^{36}$.

Embora os teores das frações P-Fe e P-Acet. tenham sido maiores na segunda campanha, o percentual de partição dessas frações inorgânicas não variou significativamente entre as duas campanhas; entretanto, na campanha de 2003, foi identificado um incremento no percentual médio da fração P-Detr. em relação à campanha anterior. Nesta ocasião o teor médio de P-Detr. em relação ao PT dobrou comparativamente à campanha anterior, atingindo $12,5 \%$, enquanto em relação ao PIT aumentou para cerca de $16 \%$. Ou seja, as condições hidroquímicas favoreceram ao aumento da deposição da fração detrítica (P-Detr.), ou houve uma maior entrada desta fração geoquímica de fósforo que corresponde a fósforo associado a material lixiviado dos solos com o aumento das entradas de fósforo total. Houve correlação significativa da fração de fósforo detrítico com o ferro $(\mathrm{r}=0,756 ; \mathrm{n}=6, \alpha=0,05)$, mostrando que o ambiente estava favorável às precipitações desta fração.

$\mathrm{O}$ percentual da fase detrítica em relação às demais frações inorgânicas refletiu o baixo fluxo de sedimentos continentais para o estuário e caracterizou a região de influência fluvial no estuário, confirmando que o grande número de barramentos ao longo da bacia hidrográfica do Rio Jaguaribe promove a retenção de água, sedimentos e nutrientes ${ }^{25}$. Desta forma a extensão das deposições sedimentares de P-Detr. resume-se à porção mais continental do estuário (P6 a P10), sendo discreta ou não detectável sua presença em direção a foz, provavelmente só ocorrendo devido à ressuspensão provocada pela ação das marés ${ }^{14}$.

O baixo percentual da fração detrítica de fósforo em relação às demais mostrou que o aumento da fração inorgânica no estuário do Jaguaribe (CE) não está associada a aumento das emissões naturais de fósforo, corroborando a hipótese de aumento das emissões antrópicas $^{37}$, que são predominantemente compostas de ortofosfato e seus derivados inorgânicos ${ }^{9,10}$.

\section{CONCLUSÕES}

As concentrações de fósforo total, inorgânico e orgânico total nos sedimentos do estuário do Rio Jaguaribe apresentam-se dentro da faixa relatada para outros ambientes marinhos carbonáticos não impactados, como o nordeste da Baía da Florida ${ }^{19}$ e a Baía das Baleias nas Bermudas ${ }^{12}$, ambientes onde a produtividade primária é limitada pelos teores de fósforo em suas águas.

Os resultados demonstram que embora não tenha havido, durante o período avaliado, aporte diferenciado dos teores de fósforo total para os sedimentos estuarinos houve um aumento do percentual da fração inorgânica em relação ao teor de fósforo total nos sedimentos do canal estuarino, atingindo percentuais superiores a $86 \%$.
Embora a costa nordeste oriental seja conhecida como oligotrófica, os resultados deste trabalho mostram que particularmente regiões estuarinas, como a do Rio Jaguaribe (CE), podem acumular fósforo inorgânico em sedimentos estuarinos, visto que o aumento desse aporte, no caso exemplificado, não foi metabolizado pela atividade biológica local. Este fato se deve aos baixos deflúvios dos rios do semi-árido e ao elevado nível de açudagem dos rios que gera, durante as secas, retenção hídrica prolongada de águas estuarinas, somados ao aumento do aporte antrópico de fósforo inorgânico.

Para detalhamento do acúmulo da fração inorgânica de fósforo em sedimentos e conseqüente possível aumento do estado trófico do estuário, estudos focados na hidroquímica e produtividade estuarina estão sendo realizados, principalmente em áreas onde atividades que despejam efluentes enriquecidos em nutrientes estejam em franca expansão, como áreas de aqüicultura ou de urbanização sem esgotamento sanitário adequado ${ }^{38}$.

As condições ambientais atuais do estuário do Rio Jaguaribe favorecem a precipitação principalmente de formas férricas e carbonáticas do fósforo, possivelmente devido às condições físicoquímicas de suas águas, que aparentemente contribuem para o sequiestro de fósforo da coluna d'água e sua deposição nos sedimentos. Entretanto, com o possível avanço do estado trófico do estuário, é possível que esta imobilização não ocorra e o fósforo inorgânico permaneça em solução, favorecendo ainda mais o aumento da trofia do estuário ${ }^{39}$.

Os resultados obtidos para o fósforo detrítico confirmam que a contribuição desta fração para este estuário da costa nordeste oriental representa uma forma de fósforo essencialmente fluvial.

$\mathrm{O}$ esquema de extração de Berner e Rao ${ }^{7}$ permitiu a separação de seis frações geoquímicas distintas de fósforo, delimitou a influência fluvial no estuário do rio Jaguaribe e demonstrou a influência antrópica das emissões de fósforo para o estuário.

Embora a região estuarina do Rio Jaguaribe (CE) ainda apresente teores de fósforo total (PT) similares a de regiões não contaminadas, a extração de Berner e $\mathrm{Rao}^{7}$ mostrou sensibilidade analítica adequada para avaliações da qualidade ambiental de estuários da costa nordeste oriental. Estudos similares em outras regiões costeiras tropicais podem estender a aplicabilidade do uso dos resultados desta extração como indicadores ambientais.

Recentemente foi utilizada metodologia semelhante a apresentada neste trabalho para a avaliação do impacto das entradas de fósforo para ecossistema fluvial fortemente impactado pela presença da cidade de Deli (Índia) que gera 6500 t de resíduos sólidos e 2270 × $10^{6} \mathrm{~L}$ de resíduos líquidos por $\mathrm{dia}^{40}$. Da mesma forma que neste trabalho, os autores que avaliaram as frações geoquímicas de fósforo na bacia de drenagem do Ganges obtiveram resultados concludentes sobre a origem das emissões de fósforo na região e sobre a qualidade dos sedimentos estudados, ressaltando-se que naquela região os teores de PT atingiram a faixa de 2160 a $4290 \mathrm{mg} \mathrm{kg}^{-1}$.

Comparando-se os resultados da bacia do Ganges ${ }^{40}$ aos da região oligotrófica do estuário do rio Jaguaribe (CE), pode-se concluir pela ampla faixa de aplicabilidade do uso das frações geoquímicas de fósforo na avaliação de sedimentos costeiros e fluviais, caracterizando a partição geoquímica de fósforo como excelente indicador de qualidade ambiental.

\section{AGRADECIMENTOS}

Ao CNPq pela bolsa de produtividade em pesquisa de R. V. Marins, Processo 500842/2003-5, e à Funcap (CE) pela bolsa de MSc de F. J. Paula Filho. 


\section{REFERÊNCIAS}

1. Nürnberg, G.; Peters, R. H.; Berhand. Intern. Verein. Limnol. 1984, 22, 190.

2. Aminot, A.; Guillaud J. F.; Andrieux, L. F.; Oceanol. Acta 1993, 16, 617.

3. Boyd, C. E.; Laurence, M.; Aquacult. Engineer. 1999, 20, 113.

4. Marins, R. V.; Paula Filho, F. J.; Maia, S. R. R.; Lacerda, L. D.; Marques, W. S.; Quim. Nova 2004, 27, 763.

5. Dyer, K. R.; Estuaries: A Physical Introduction, $1^{\text {st }}$ ed., Wiley: New York, 1997.

6. Froelich, P. N.; Bender, M. L.; Luedtke, N. A.; Heath, G. H.; Devries, T.; Am. J. Sci. 1982, 282, 474.

7. Berner, R. A.; Rao, J. L.; Geochim. Cosmochim. Acta 1994, 58, 2333.

8. Esteves, F. A.; Fundamentos de Limnologia, $2^{\mathrm{a}}$ ed., Interciência: Rio de Janeiro, 1998.

9. Ruttenberg, K. C.; Limnol. Oceanogr. 1992, 37, 1460.

10. Osorio, V. K. L.; Oliveira, W.; Quim. Nova 2001, 24, 700.

11. Silva, C. A. R.; Tese de Doutorado, Universidade Federal de São Carlos, Brasil, 1990.

12. Jensen, H. S.; Mcglathery, K. J.; Marino, R.; Howarth, R. W.; Limnol. Oceanogr. 1998, 43, 799.

13. Mozeto, A. A.; Silvério, P. F.; Soares, A.; Sci. Total Environ. 2001, 266, 135 .

14. Marins, R. V.; Lacerda, L. D.; Abreu, I. M.; Dias, F. J. S.; Ciência Hoje 2003, 33, 66

15. Boström, B.; Persson, G.; Broberg, B.; Hydrobiologia 1988, 170, 133.

16. Ruttenberg, K. C.; Berner, R. A.; Geochim. Cosmochim. Acta 1993, 57, 991.

17. Ingall, E; Jahnke, R.; Mar Geol. 1997, 139, 219

18. Sherman, R. E.; Fahey, T. J.; Howarth, R. W.; Oecologia 1998, 115, 553.

19. Koch, M. S.; Benz, R. E.; Rudnick, D. T.; Estuar. Coast. Shelf Sci. 2001, $52,279$.

20. Marins, R. V.; Dias, F. J. S.; Anais do IX Congresso Brasileiro de Geoquímica, Belém, Brasil, 2003.

21. Dias, F. J. S.; Marins, R. V.; Maia, L. P.; Anais do X Congresso Brasileiro de Geoquímica, Porto Galinhas, Brasil, 2005.
22. IPLANCE, Instituto de Planejamento do Ceará; Ranking dos Municípios, Imprensa Oficial do Estado do Ceará: Fortaleza, 1997.

23. Abreu, I. M.; Lacerda, L. D.; Marins, R. V.; Anais do VI Congresso de Ecologia do Brasil, Fortaleza, Brasil, 2003.

24. Maia, L. P.; Dissertação de Mestrado, Universidade Federal de Pernambuco, Brasil, 1989

25. Marins, R. V.; Freire, G. S. S.; Maia, L. P.; Lima, J. P. R.; Lacerda, L. D. Em South American Basins - LOICZ Reports \& Studies. 21; Lacerda, L. D.; Kremer, H. H.; Salomons, W.; Crossland, J. C., eds.;LOICZ-IGBP: Texel, 2002, cap. 7.

26. Hansen, H.P; Koroleff, F. Em Methods of Seawater Analysis; Grasshoff, K.; Kremling, K.; Ehrhardt, M., eds.; $2^{\text {nd }}$ ed., Wiley: New York, 1999, cap. 10 .

27. Forstner, U.; Salomons, W.; Metals in the Hydrocycle, Springer: Berlin, 1980.

28. Psenner, R.; Boström, B.; Dinka, M.; Petterson, K.; Puesko, R.; Sager, M.; Arch. Hydrobiol. 1988, 30, 98 .

29. Stone, M.; English, M. C.; Hydrobiologia 1993, 253, 17.

30. Golterman, H. L.; Hydrobiologia 1996, 335, 87.

31. Fabre, A.; Fromard, F. R.; Trichon, V.; Hydrobiologia 1999, 392, 13.

32. Watanabe, F. S.; Olsen, S. R.; Soil Sci. 1962, 93, 183.

33. Loring, D. H.; Rantala, R. T. T.; Earth Sci. Rev. 1992, 32, 235.

34. Salomons, W.; Stigliani, W. M.; Biogeodynamics of Pollutants in Soils and Sediments, Springer: Berlin, 1995.

35. Miller, L. C.; Miller, J. N.; Statistics for Analytical Chemistry, Ellis Horwood and Prentice Hall: London, 1994.

36. Andrieux, L. F.; Aminot, A.; Estuar. Coast. Shelf Sci. 2001, 52, 617.

37. Santos, J. A.; Dissertação de Mestrado, Universidade Federal do Ceará, Brasil, 2005.

38. Eschrique, S. A.; Dissetação de Mestrado, Universidade Federal do Ceará, Brasil, 2007.

39. Paula Filho, F. J.; Dissertação de Mestrado, Universidade Federal do Ceará, Brasil, 2004.

40. Moturi, M. C. Z.; Rawat, M.; Subramanian, V.; Chemosphere 2005, 60, 237. 